\title{
A STUDY ON THE PLIED COTTON RING SPUN YARN TENACITY
}

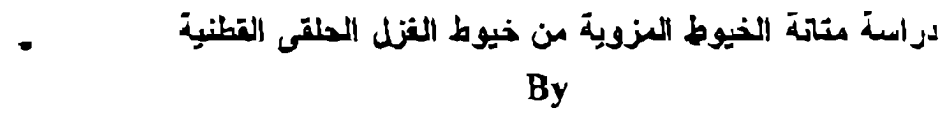

Assoc. Prof. I. M. Rakha

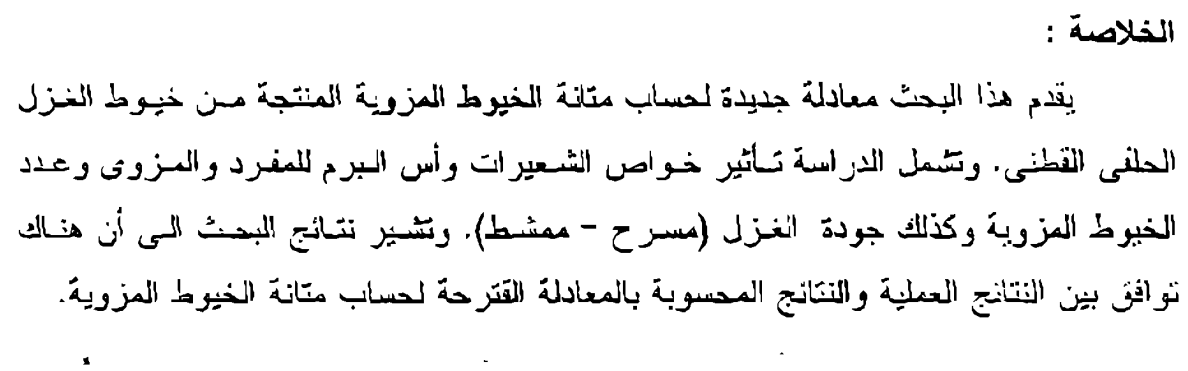

Abstract :

This work presents an emperical formula of plied yan strength produced from ring spun cotton yarns. Expeniments carried out on several samples of cotton fibres and the investigation concemed with the influence of fibre properties, single twist, ply twist and number of plies and spimuing quality.

The results obtained with this fornula agree satisfactonly with the experinental data.

\section{(1) Introduction :}

The strength of plied staple yarns vary complicatedly according to the fibre properties, number of plies, twist directions and single and ply twist multipliers.

Expenimental studies on this subject has been carried out $11,2,3,4,5,6,8 /$ but not considered enough investigation from the point of view of analytical treatment. On the other hand there are inany analytical researchers $2,7,9,10,11,12,13,14,15, /$ but the proposed equation deduced for predicting the strength of plied staple yams is not accurate enough because there are no equation taking into consideration all the factors affecting plied yarn strength.

The aim of the present study is predicting a formula for determination the strength of plied cotton yarns considering the following parameters : fiber parameters such as : "Fibre strength, length and tineness", single, ply yarn twist, number of plies and twist diction. 


\section{(2) Theoretical analysis :}

The literature suggested that yam tensile properties were affected by soveral parameters such as tore strength, strength irregularity, perentage of broken fibres and yam twist. What follows is a trial inodifying these facts. The determination of plied yam strength it is important to consider that following parameters :

1) The effect of plied yam strength irregularity:

From the theory of folding, it is known the yam irregularity of cross section and consequently the strength irregularity decreases as the number of plies increases, and theoretically the following formula can be used.

$$
\mathrm{V}=\frac{100}{\sqrt{\mathrm{m}_{0} \mathrm{~m}_{1}}}=\frac{\sigma}{\overline{\mathrm{p}}} 100 \approx 1.25 \mathrm{U}
$$

Where: $\mathrm{V}$ : Coefficient of variation of yam strength.

mo : Number of fibres in single yam cross-section.

$m_{1}:$ Number of plies.

$\sigma:$ The standard deviation of strength.

$\bar{p}$ : The average strength of the yam.

$U$ : Linear irregularity of single yam strength $/ 6 /$, and equal to

$$
=\mathrm{U}_{0}+\frac{65}{\sqrt{\mathrm{m}_{0}}}
$$

Where $: U_{0}=$ Coefficient depends on the spinning quality and equal to $: 3$ for combed yams and 4.5 for carded yams.

By combining equations (1) and (2) we obtain :

$$
\mathrm{U}_{1}=\frac{\mathrm{U}_{0}}{\sqrt{\mathrm{m}_{1}}}+\frac{65}{\sqrt{\mathrm{m}_{0} \mathrm{~m}_{1}}}
$$

It is also known that the yam breakage at very week points, and consequently the regarding value of tensile tester can be calculated as follows

$$
\mathrm{p}=\overline{\mathrm{p}}-3 \sigma
$$

Where: $\quad P$ : tensile strength reading.

By substituting eq. (1) and (3) in (4), we obtain :

$$
\mathrm{p}=\overline{\mathrm{p}}\left(1-\frac{0.0375}{\sqrt{\mathrm{m}_{\mathrm{l}}}} \mathrm{U}_{0}-\frac{2.44}{\sqrt{\mathrm{m}_{0} \mathrm{~m}_{1}}}\right)
$$

The term between brackets defined physically as the uniformity coefficient of plied yam strength.

The experimental results of strength irregularity of plied y;ms and those calculated by formula (3) are shown in table (4) 


\section{(2) The percentage of broken fibres :}

From the earlier studies $17,9,16 /$ concerned with the behavior of spun yams during breakage and the mechanics of strength of the plied yam it was found that the pressure along the fibre in spun yans increases from zero to the value determined by the yarn extension and then decreased to zero at the other end. If we assume that the frictional force which prevents fibre slippage created on a length of fibre equal $1_{\mathrm{S}} 1$, thus the fibres presents in the yarn and firmly gripped with the meighbours fibres at length longer than $I_{\mathrm{S}}$ will break and the other fibres which has a contact length less than $1_{S l}$ will be subjected to slippage. Thus the percentage of broken fibres which contributed in the yarn strength are equal to

$$
\left(1-\frac{2 L_{\text {st }}}{L_{\text {st. }}}\right) / 17 \text {. }
$$

Where: $1_{\text {st. }}$ : Staple length of fibres.

Also from the theoretical assumptions given by Varasheelaf 11 it is found that; the forces acting on the fibre element of the yarn as shown in Fig. (1) represented by the following relation :

$$
\mathrm{dN}=2 \mathrm{Y} \sin \frac{\mathrm{d} \phi}{2}-\mathrm{dy} \sin \frac{\mathrm{d} \phi}{2}+\rho \sigma \mathrm{d} \phi
$$

Where : $\quad \mathrm{dN}$ : The reaction on the fibre element.

$Y$ : The tension on the fibre element.

$\rho$ : The radius of twist curvature of the plain in which the fibre lies.

$\sigma:$ The stress from the onter layers on the unit length.

It was considered that $\sin \mathrm{d} \phi=\mathrm{d} \phi$ and the snnall values are negligent. Equation (6) can be rewritten as follows :

$$
\mathrm{dN}=\mathrm{Yd} \phi+\rho \sigma \mathrm{d} \phi
$$

Also the value of dy taken from the projection of the tangent as follows :

Where: $\quad \mathrm{H}=$ Fibre griping force.

$\mathrm{f}=$ Coefficient of friction between fibres.

Equation (7) can be applied for plied yarn by neglecting $H$, and equal to

From equations (6) and (7) we can get

$$
\mathrm{dy}=\mathrm{fd} \mathrm{N}
$$

$$
\mathrm{d} \phi=\frac{\mathrm{dy}}{\mathrm{FY}+\mathrm{F} \rho \sigma}
$$

By integrating $d \phi$ 
Where: $\quad Y=P_{f}$ : Fibre breaking load.

$$
\phi_{s t}=\int_{0}^{y} d=\int_{0}^{y} \frac{d y}{f y+f}=\frac{1}{f} \ln \frac{f y+f}{f}
$$

$$
\begin{aligned}
& \phi_{\text {sl. }}=\int_{0}^{L_{L t}} \frac{d \mathrm{t}}{\rho}=\frac{L_{s \mathrm{~s}}}{\rho} \\
& \rho=\frac{\mathrm{r}}{\sin ^{2} \beta}=\text { const } .
\end{aligned}
$$

$r$ : Yanı radius.

$\beta$ : Twisting angle.

From the previous equations $(9),(10)$ and (11), we can get :

$$
\mathrm{L}_{\mathrm{sL}}=\phi_{\mathrm{s \omega}} \rho=\frac{\rho}{\mathrm{f}} \ln \left(1+\frac{\mathrm{P}_{\mathrm{i}}}{\rho \sigma}\right)
$$

Where: $\quad \sigma: \mathrm{P}_{f} / 2 \rho$ from varsheelaf /11/

since the twist spiral radius is not actually equal to the yarn radius as stated in the previous work $/ 18 / \mathrm{d}_{\mathrm{s}}=0.8 \mathrm{~d}$.

Where: $d_{s}$ : The diameter of twist spiral.

$\mathrm{d}$ : The actual yarn diameter.

Consequently, it is recommended to take $\sigma=\frac{P_{i}}{1.6}$

$$
\mathrm{L}_{\mathrm{sl} .}=\frac{\rho}{\mathrm{f}} \ln 2.6=\frac{0.5 \mathrm{~d}_{\mathrm{s}}}{\mathrm{F} \sin ^{2} \beta}=\frac{0.4 \mathrm{~d}}{\mathrm{~F} \sin ^{2} \beta}
$$

Where : $\quad \mathrm{F}=0.25-\frac{0.006 \mathrm{p}_{\mathrm{f}}}{\rho}$

(15) varasheelf $/ 11 /$, and

$$
\mathrm{F}=\frac{2}{2+\sqrt{\mathrm{N}}}
$$

(16) sakaloof $/ 21 /$

Where : $\mathrm{N}$ : is the metric yarn count.

The coefficient of friction " $f$ " between fibres is not constant. In relation with twist it is linearly proportional with " $\alpha_{T}$ " as given by the following relation $/ 19 /$

$$
F=\frac{\alpha_{\mathrm{T}} \cdot 10^{-2}}{\sqrt{\alpha_{\mathrm{T}} \cdot 10^{-2}}+30}
$$

Where : $\quad \alpha_{T}$ : is the twist multiplier of plied yam and equal to

$$
\alpha_{\mathrm{T}}=\tau_{\mathrm{p}}\left(\alpha_{\mathrm{s}} 10^{-4} /(0.8)^{\mathrm{ml} \text { in }}\right.
$$

$d_{p}$ :is the plied yam diameter and equal to : $d_{s}, C$ 
Where : $\mathrm{C}$ : Constant depends on the number of plies, the value $\mathrm{C}$ recominended by sakaloof $/ 21 /$ is given in table (3).

\begin{tabular}{|c|c|c|c|c|c|}
\hline $\mathrm{ml}$ & 2 & 3 & 4 & 5 & 6 \\
\hline $\mathrm{C}$ & 1.8 & 2.2 & 2.56 & 2.86 & 3.13 \\
\hline
\end{tabular}

$\beta:$ is the twisting angle and equal to $\tan ^{-1} \pi d_{p} \tau_{p}$

Where : $\tau_{p}$ : Turms per meter of plied yarn.

It is roughly known that the mean yarn strength $p$ equal to

$$
\mathrm{P}_{\mathrm{f}}, \mathrm{m}_{0} \mathrm{~m}_{1}\left(1-\frac{2 \mathrm{~L}_{\mathrm{sl}}}{\mathrm{L}_{\mathrm{st} .}}\right) \mathrm{x}
$$

Where $\mathrm{x}$ : is the twist correction factor.

By combining equation (20) and (5) we can get :

$$
\mathrm{P}=\mathrm{P}_{\mathrm{i}} \mathrm{m}_{0} \mathrm{~m}_{1}\left(1-0.0375 \frac{\mathrm{U}_{0}}{\sqrt{\mathrm{m}_{1}}}-\frac{2.44}{\sqrt{\mathrm{m}_{0} \mathrm{~m}_{1}}}\right)\left(1-\frac{2 \mathrm{~L}_{\text {st }}}{\mathrm{L}_{\text {st. }}}\right) \mathrm{x}
$$

\section{(3) The twist correction factor :}

In case of single yarns it is easy to take the correction factor equal to 1 when the yam twist factor reaches its oprimum value, and then decreases according to the difference between the optinuin and actual twist factors. But in case of plied yam the problem becomes more complicated, because there are three factors must be taken into account, these are $\left(\alpha_{\mathrm{S}}\right)$ single twist factor, $\left(\mathrm{m}_{\mathrm{l}}\right)$ number of plies and ply twist factor $\left(\alpha_{p}\right)$

$$
\text { Thus } X_{1}=2.55\left(\frac{m_{1}}{\alpha_{s}}\right)^{0.14}
$$

According to the nature of strength, twist curves for different number of plies the value $m_{1}$ corresponding six plies is equal to 2.5 .

While the value of twist correction factor $\mathrm{X}_{2}$, as slown in table (3), depends on the difference between the actual twist factor and optimum twist factor $/ 20 /$

Thus equation (21) can be rewritten as follows :

$$
\mathrm{P}=\mathrm{P}_{\mathrm{r}} \mathrm{m}_{0} \mathrm{~m}_{1}\left(\frac{1-0.0375}{\sqrt{\mathrm{ml}}}-\frac{2.44}{\sqrt{\mathrm{m}_{0} \mathrm{~m}_{1}}}\right)\left(1-\frac{2 \mathrm{~L}_{\mathrm{sl}}}{\mathrm{L}_{\mathrm{st}}}\right) \mathrm{x}_{1} \mathrm{x}_{2}
$$

\section{(3) Experimental work :}

To verify the plied strength predicted from the formula deduced in the present work, a sample of soviet cottons were selected to reduce single ring spun yams having different linear densities at different levels of twist factors. 
These yams plied in $\mathrm{S}$ direction with different number $\mathrm{f}$ plies ranges from 2 to 6 at different twist multipliers.

A controlled sample from.Egyptian cotton G-77 was spun into single and plied yams.

The fibre properties are shown in table (1) and the plied yarus produced shown in table (4).

The fibre properties are shown in table (1) and the plied yarns produced shown in table (4).

The produced yams were tested for strength characteristics, strength (gn/tex), C.V\% of strength, yam twist and yam linear density.

\section{(4) Results and Discussions :}

From the experimental results shows in table (4), it is clear that, the strength of plied yam increases as the number of plies increases from 2 to 5 and then decreases as the number $\delta$ plies increases. This can be attributed to the components arrangement imply yam cross-section, because single components taking a pipe form in regular pattern and permits equal pressure in each coinponent and results in a higher strength of plied yarm. On the other hand, with higher number of plies one or more components tend to be in the yam core while the others wrapping around them. This situation causes non regular pressure in the components and consequently reduces plied yam strength. Also the plied yarn strength was affected by the amount of single and ply twist and twist directions. The results slows a ligher strength for plied yam produced from single with lower initial twist than those obtained with higher single twist.

This was explamed by the increment in the pressure generated by ply twist which over comes the decrement in single pressure as a result of untwisting it with small values for yarns having small twist in singles, and consequently such yarns introduced high pressure values reaches the optimum, strength values. But in case of single yarns with a high ply twist multiplier the decrement in pressure coming from the decrease in single twist which exceeds an increase by ply twist, and strength increases, the increase in pressure by ply twist gradually assumes large proportions and eventually rises above critical level. The experimental results is in agreement with the earlier studies $/ 1,2,3,4,5,6,8$.

The experimental results were compared to calculated values of plied yarn strength for all tested cotton samples.

As shown in table (4) and Fig. (2), which represents the results of the Egyptian cotton sample, there is a slight difference between the actual and predicted values of plied yam strength.

A computer programs was developed to fit the experimental data of equation (23) 


\section{(5) Example of calculation :}

In order to produce a sewing threads of $70 / 3 \mathrm{Ne}$ from Egyptian cotton sample No. (3), (Table I)

A single yarn of 8.4 tex $(70 \mathrm{Ne})$ were spun at twist factor equal 0.8 $\alpha_{s_{\mathrm{st}}}=3400 * 0.8=2720 \alpha_{\mathrm{T}_{\mathrm{d}}} / 22 /$

By substituting in $\left(1-0.0375 \frac{U_{0}}{\sqrt{m_{1}}}-\frac{2.44}{\sqrt{m_{0} m_{1}}}\right)$, where $m_{l}=3, U_{0}=3$ (combed) and $m_{\mathrm{O}}=69.4$ fibres, thus the value between brackets equal 0.77 .

$$
\mathrm{L}_{\mathrm{sl} .}=\frac{0.4 \mathrm{~d}}{\sin ^{2} \mathrm{~B}} \quad \text { where } ; \mathrm{d}=\mathrm{d}_{\mathrm{s}} \mathrm{C}, \mathrm{C}=2.2 \text { (from table "2") }
$$

and $d_{S}=0.0819 \frac{T_{s}^{1.18}}{\left(\alpha_{s} 10^{-2}\right)^{0.54}}=0.118 \mathrm{~mm} / 18 /$.

$\beta=\tan ^{-1} \pi . d \tau_{c r}$, where $\tau_{c r}$ is the critical T.P.M. of plied yam corresponding critical twist factor of plied yarn which equal :

$3660 \alpha_{\Upsilon}$, then by substituting in equation (18) we can get $\tau_{\mathrm{cr}}=1294$

T.P.M. and $F=36.6 .1 \sqrt{36.3}+30=0.20$

Thus $\left(1-\frac{2 \mathrm{~L}_{\mathrm{sl}}}{\mathrm{L}_{\mathrm{si}}}\right)=\left(\frac{1.96}{36.5}\right)=0.95$

* The correction factor of twist

$$
x_{1}=2.55\left(\frac{m_{1}}{\alpha_{s}}\right)^{0.14}=2.55\left(\frac{3}{2720}\right)^{1.44}=0.98
$$

* The correction factor of twist $\mathrm{X}_{2}=1\left(\alpha_{\mathrm{cr}}\right)$.

* From table (1): $P_{f}=4.8 \mathrm{gm}$ and $1_{s t}=36.5 \mathrm{~mm}$.

* By substitution in equation (23), we can get : $\mathrm{P}=4.8 * 69.4 * 3 * 0.77 * 0.95 * 0.98=716.4 \mathrm{gm}=28.4 \mathrm{~g} / \mathrm{tex}$

\section{(6) Conclusions :}

The study of plied yarn strength characteristics gives the following conclusions :

(1) The study affords a formula to predict the strength of cotton plied yams.

(2) The suggested formula clearly show, the piied yarn strength dependent on fibre properties, single and ply twist, number of plies and spinning quality.

(3) The calculated values deduced from the suggested formula agree satisfactorily with the experimental results 


\section{References :}

(1) S. Kershaw ; J. Text. Inst. ; 27, 1936

(2) A.F.W. Eoulson, Dakin, J. Text. Inst:, 48, 1957.

(3) L.A. Fion, J.J. Brown, J.E. Sand, Text. Res. J. 24, 1954.

(4) J.F. Bogdan, Text. Res. J., 26, 1956.

(5) K.E. Karetski, Nautshna - Esledavateısky Trudy, TSENKHBE, Gezlegpram, 1958.

(6) A.N. Salavuf, Nautshna - Esledevetlsky Trudy, TSENKHB, GEZLEGPROM, 1958.

(7) H. Kyuma, M. Kobayashi and T. Kazama ; J. Text Mach. Soc. Japan, Vol. 16, No. 5, 1970.

(8) M.M. Platt, Text. Res-J., 24, 2, 1954.

(9) E.R. Schwarz, J. Text. Inst. March, 1933

(10) Kyuma, J. Text. Moch. Soc. Japan, 13, 305, 960.

(11) V.A. Varasheelaf, textelnaia Promeshlensot, No. 3, 1946 ; No. 9 , 1946; No. 7, 1947; No. 11, 1948; No. 1, 1949.

(12) K.U. Karetski, Krootelnoy E. Neetelshony Prezvodstva, Gezlegprom, 1957.

(13) K.U. Karetski, Asnovy Praikteravania Svoistv Priagy. Moscow, 1962.

(1.4) K.U. Karetski, Engineery Praikteravania Textelneklı Materialav. Moscow, 1971.

(15) V.A. varasheelaf, Sobrnech Rabot Evanoveckova Texteelanva Instinta, 1947

(16) J.W. Hearle et al., "Structural Mechanics of Fibres, Yanns and Fabrics" Vol. 1, Wiley Intersciecne.

(17) M.A. EL-Messiry, The Bulletin of the Faculty of Engimening, Alexandria Univ. Vol. XIX, No. 1, 1980.

(18) I. Rakha and R. EL-Bealy, MEJ, Vol. 18, No. 4, Dec. 1993.

(19) Rakha J., A study of the strength of ring spun yarns, Teklunalogia Textelnoy Promushlenosty, Accepted for Rub.

(20) I. Rakha, R. EL-Bealy and M. EL-Messiry, MEJ, Vol. 20, No. I March, 1995.

(21) G.V. Sakalov, Teoria Krotshenia Valaknestekh Materialav, Moscow, 1977.

(22) Rakha I.M. Tekhnologia textelnoy Promushlenosy, No. 5, 1993. 
Table (1): Physical and Mechanical properies of cotton fibres

\begin{tabular}{|c|c|c|c|c|}
\hline $\begin{array}{c}\text { Staple length } \\
\mathbf{m m}\end{array}$ & $\begin{array}{c}\text { Linear dengity } \\
\text { tex }\end{array}$ & $\begin{array}{c}\text { Breaking logd } \\
\mathbf{g m}\end{array}$ & $\begin{array}{c}\text { Ten:acity } \\
\text { gm/tex }\end{array}$ & $\begin{array}{c}\text { Single count produced } \\
\text { tex }\end{array}$ \\
\hline 32.7 & 0.183 & 4.2 & 22.95 & 25 (carded) \\
40.2 & 0.135 & 4.4 & 32.59 & $10,12,15$ (combed) \\
36.5 & 0.121 & 4.8 & 39.7 & 12 (combed) \\
\hline
\end{tabular}

Table (3): The correction rwist factor $\mathrm{X}_{2}$

\begin{tabular}{|c|c|c|c|c|c|c|c|c|c|}
\hline$\alpha_{T-\alpha_{c r}}$ & -1660 & -1380 & -1100 & -830 & -550 & -280 & -140 & 0 & 140 \\
\hline$X_{2}$ & 0.8 & 0.835 & 0.87 & 0.9 & 0.935 & 0.97 & 0.985 & 1 & 0.98 \\
\hline$\alpha_{T-\alpha_{c r}}$ & 370 & 740 & 1110 & 1480 & 1860 & 2200 & 2600 & 3000 & 3.340 \\
\hline$X_{2}$ & 0965 & 0.93 & 0.805 & 0.865 & 0.83 & 0.8 & 0.765 & 0.73 & 0.7 \\
\hline
\end{tabular}

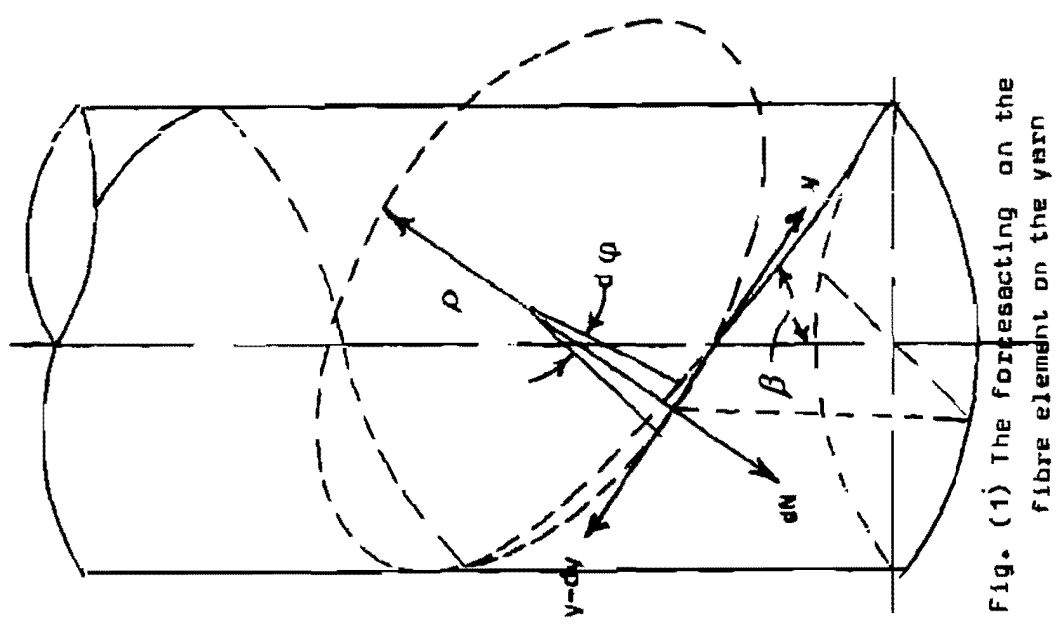




\begin{tabular}{|c|c|c|c|c|c|c|c|c|c|c|c|c|c|}
\hline $\begin{array}{l}\text { Yam } \\
\text { counit } \\
\text { (1ex) }\end{array}$ & $\begin{array}{l}\text { Single } \\
\text { ivist } \\
\text { fitclor }\end{array}$ & $\begin{array}{c}\text { ply } \\
\text { livist } \\
\text { fictor }\end{array}$ & $\begin{array}{c}\text { Exp. } \\
\text { lenocily } \\
\text { g/lex }\end{array}$ & $\begin{array}{c}\text { cal. } \\
\text { Ienacily } \\
\text { g/lex }\end{array}$ & $\begin{array}{l}\text { Exp. } \\
\text { U\% }\end{array}$ & $\begin{array}{l}\text { Cal. } \\
U \%\end{array}$ & $\begin{array}{c}\text { Yara } \\
\text { comat ex }\end{array}$ & $\begin{array}{l}\text { SIngle } \\
\text { civist } \\
\text { factur }\end{array}$ & $\begin{array}{l}\text { Myy } \\
\text { ivise } \\
\text { faclor }\end{array}$ & $\begin{array}{c}\text { Exp. } \\
\text { tenacily } \\
\text { g/lex }\end{array}$ & $\begin{array}{l}\text { call. } \\
\text { lenacily } \\
\text { g/lex }\end{array}$ & $\begin{array}{l}\text { Exp. } \\
\text { U\% }\end{array}$ & $\begin{array}{l}\text { Cal. } \\
\text { U\% }\end{array}$ \\
\hline $9.9 \times 2$ & 3101 & $\begin{array}{l}1940 \\
2291 \\
3003 \\
3559 \\
3902\end{array}$ & $\begin{array}{l}16.0 \\
17.3 \\
189 \\
19.6 \\
19.0\end{array}$ & $\begin{array}{l}14.6 \\
16.0 \\
18.5 \\
202 \\
199\end{array}$ & 7.8 & $\overline{7.5}$ & $1243 \times 3$ & 3779 & $\begin{array}{l}2606 \\
3318 \\
4002 \\
4513 \\
5161 \\
\end{array}$ & $\begin{array}{l}197 \\
21.5 \\
216 \\
21.0 \\
20.5 \\
\end{array}$ & $\begin{array}{l}17.0 \\
20.4 \\
21.7 \\
20.7 \\
19.8 \\
\end{array}$ & 5.8 & . \\
\hline $9.9 \times 3$ & 3101 & $\begin{array}{l}2610 \\
2923 \\
3308 \\
4064 \\
4642\end{array}$ & $\begin{array}{l}17.3 \\
181 \\
19.7 \\
19.8 \\
18.1\end{array}$ & $\begin{array}{l}18.9 \\
202 \\
21.0 \\
22.2 \\
21.2\end{array}$ & 6.3 & 6.1 & $12.43 \times 4$ & $\overline{3779}$ & $\begin{array}{l}2745 \\
3493 \\
1004 \\
259 \\
5568\end{array}$ & $\begin{array}{l}19.8 \\
22.6 \\
227 \\
22.0 \\
20.6\end{array}$ & $\begin{array}{l}19.1 \\
22.7 \\
23.0 \\
21.6 \\
20.0\end{array}$ & 5.1 & 49 \\
\hline $9.9 \times \overline{4}$ & 3101 & $\begin{array}{l}2469 \\
2653 \\
3130 \\
3677 \\
4253\end{array}$ & $\begin{array}{l}200 \\
21.6 \\
217 \\
21.9 \\
20.9\end{array}$ & $\begin{array}{l}19.1 \\
22.1 \\
24.1 \\
24.3 \\
23.2\end{array}$ & 52 & $5 . \overline{3}$ & $\overline{12} 4 \overline{3 \times 5}$ & 3779 & $\begin{array}{l}2548 \\
3177 \\
3624 \\
4265 \\
5271\end{array}$ & $\begin{array}{l}20.0 \\
224 \\
23.1 \\
226 \\
215\end{array}$ & $\begin{array}{l}178 \\
218 \\
23.9 \\
22.8 \\
21.4 \\
\end{array}$ & 4.2 & 4.4 \\
\hline $99 \times 5$ & 3101 & $\begin{array}{l}2682 \\
3160 \\
3678 \\
4181 \\
4650 \\
\end{array}$ & $\begin{array}{l}225 \\
22.9 \\
23.1 \\
21.5 \\
200\end{array}$ & $\begin{array}{l}20.2 \\
22.2 \\
24.5 \\
23.4 \\
22.5\end{array}$ & 4.9 & 4.7 & $12.43 \times 6$ & 3779 & $\begin{array}{l}2884 \\
3276 \\
4142 \\
4815 \\
6.937 \\
\end{array}$ & $\begin{array}{l}200 \\
20.3 \\
20.1 \\
19.5 \\
18.8\end{array}$ & $\begin{array}{l}17.7 \\
202 \\
21.2 \\
20.7 \\
16.8 \\
\end{array}$ & $3 \overline{9}$ & $4 . \overline{0}$ \\
\hline $99 \times 6$ & $3 \overline{101}$ & $\begin{array}{l}2686 \\
3048 \\
3555 \\
4135 \\
4597\end{array}$ & $\begin{array}{l}204 \\
20.6 \\
20.9 \\
20.0 \\
19.4\end{array}$ & $\begin{array}{l}18.9 \\
206 \\
22.8 \\
22.7 \\
21.8\end{array}$ & 4.2 & 4.3 & $11.65 \times 3$ & 2980 & $\begin{array}{l}2024 \\
2651 \\
3179 \\
3805 \\
2029 \\
2784 \\
4013 \\
4650 \\
\end{array}$ & $\begin{array}{l}20.3 \\
20.5 \\
241 \\
23.4 \\
244 \\
269 \\
27.2 \\
260 \\
\end{array}$ & $\begin{array}{l}18.4 \\
21.5 \\
23.8 \\
253 \\
19.3 \\
243 \\
276 \\
26.3 \\
\end{array}$ & 58 & 6.8 \\
\hline
\end{tabular}




\begin{tabular}{|c|c|c|c|c|c|c|c|c|c|c|c|c|c|}
\hline $\begin{array}{c}\text { Yarn } \\
\text { count } \\
\text { (Icx) } \\
\end{array}$ & $\begin{array}{l}\text { Single } \\
\text { livist } \\
\text { faclor } \\
\end{array}$ & $\begin{array}{c}\text { ply } \\
\text { Iivisi } \\
\text { faclor } \\
\end{array}$ & $\begin{array}{c}\text { Exp. } \\
\text { Ienocity } \\
\text { g/lex }\end{array}$ & $\begin{array}{c}\text { cal. } \\
\text { tenacity } \\
\text { g/tex }\end{array}$ & $\begin{array}{l}\text { Exp. } \\
U \%\end{array}$ & $\begin{array}{l}\mathrm{Cnl} \\
\mathrm{U} \%\end{array}$ & $\begin{array}{c}\text { Yarn } \\
\text { counl tex }\end{array}$ & $\begin{array}{l}\text { SIngle } \\
\text { Iwist } \\
\text { faclor }\end{array}$ & $\begin{array}{c}\text { ply } \\
\text { 1wist } \\
\text { factor } \\
\end{array}$ & $\begin{array}{c}\text { Exp. } \\
\text { tenacily } \\
\text { g/lex }\end{array}$ & $\begin{array}{c}\text { cal. } \\
\text { renacity } \\
\text { g/lex }\end{array}$ & $\begin{array}{l}\text { Exp. } \\
\text { U\% }\end{array}$ & $\begin{array}{l}\text { Col. } \\
v \%\end{array}$ \\
\hline $11.65 \times 4$ & 2980 & $\begin{array}{l}2286 \\
2957 \\
4930 \\
\end{array}$ & $\begin{array}{l}243 \\
25.5 \\
27.2 \\
\end{array}$ & $\begin{array}{l}21.8 \\
26.2 \\
27.4 \\
\end{array}$ & 4.6 & 4.8 & $1493 \times 4$ & 3452 & $\begin{array}{r}2645 \\
3138 \\
3676 \\
\end{array}$ & $\begin{array}{l}21.2 \\
22.4 \\
229 \\
\end{array}$ & $\begin{array}{l}19.1 \\
21.7 \\
24.0 \\
\end{array}$ & 4.5 & 4.6 \\
\hline $11.65 \times 5$ & 2980 & $\begin{array}{l}2432 \\
3070 \\
4467 \\
5768 \\
\end{array}$ & $\begin{array}{l}259 \\
264 \\
279 \\
264 \\
\end{array}$ & $\begin{array}{l}22.3 \\
26.9 \\
28.5 \\
25.6 \\
\end{array}$ & 4.2 & 4.3 & $14.93 \times 5$ & 3452 & $\begin{array}{l}4591 \\
5175 \\
2768 \\
3303 \\
\end{array}$ & $\begin{array}{l}22.5 \\
218 \\
22.4 \\
23.4 \\
\end{array}$ & $\begin{array}{l}22.6 \\
21.4 \\
19.5 \\
22.1 \\
\end{array}$ & 4.1 & 41 \\
\hline $1165 \times 6$ & 2980 & $\begin{array}{l}2524 \\
3186 \\
4636 \\
5639 \\
\end{array}$ & $\begin{array}{l}24.7 \\
26.3 \\
24.9 \\
235 \\
\end{array}$ & $\begin{array}{l}21.4 \\
24.1 \\
26.6 \\
24.5 \\
\end{array}$ & 4.0 & 3.9 & $14.93 \times 6$ & 3452 & $\begin{array}{l}3871 \\
4514 \\
4595 \\
2664 \\
\end{array}$ & $\begin{array}{l}23.5 \\
22.3 \\
21.6 \\
20.5 \\
\end{array}$ & $\begin{array}{l}23.8 \\
22.9 \\
21.2 \\
18.5 \\
\end{array}$ & 3.7 & 3.8 \\
\hline $1493 \times 2$ & 3452 & $\begin{array}{l}2323 \\
2831 \\
3572 \\
3784 \\
4602 \\
\end{array}$ & $\begin{array}{l}18.6 \\
20.3 \\
20.7 \\
19.8 \\
190\end{array}$ & $\begin{array}{l}15.8 \\
18.0 \\
20.7 \\
20.4 \\
19.4 \\
\end{array}$ & 6.7 & 6.5 & & & $\begin{array}{r}3320 \\
3961 \\
+3833 \\
3775\end{array}$ & $\begin{array}{l}20.8 \\
20.4 \\
18.9 \\
17.6\end{array}$ & $\begin{array}{l}20.8 \\
22.1 \\
21.0 \\
19.4\end{array}$ & & \\
\hline $1493 \times 3$ & 3452 & $\begin{array}{l}2311 \\
2805 \\
3135 \\
3635 \\
4492 \\
\end{array}$ & $\begin{array}{l}20.3 \\
21.5 \\
218 \\
22.5 \\
210 \\
\end{array}$ & $\begin{array}{l}16.5 \\
19.2 \\
20.6 \\
22.7 \\
21.3\end{array}$ & 5.5 & 5.3 & & & & & & & \\
\hline
\end{tabular}




\begin{tabular}{|c|c|c|c|c|c|c|c|c|c|c|c|}
\hline $\begin{array}{l}\text { Yarn } \\
\text { coint } \\
\text { (tex) } \\
\end{array}$ & $\begin{array}{l}\text { Single } \\
\text { twist } \\
\text { factor } \\
\end{array}$ & $\begin{array}{l}\text { ply twist } \\
\text { factor }\end{array}$ & $\begin{array}{c}\text { Exp. } \\
\text { tenocity } \\
\text { g/tex }\end{array}$ & $\begin{array}{c}\text { cal. } \\
\text { Icnacity } \\
\text { g/tex }\end{array}$ & $\begin{array}{l}\text { Exp. } \\
\text { U\% }\end{array}$ & $\begin{array}{l}\text { Cal. } \\
\text { U\%! }\end{array}$ & $\begin{array}{c}\text { Y'rin } \\
\text { coumt tex }\end{array}$ & $\begin{array}{l}\text { SIngle } \\
\text { Iivisi } \\
\text { factor }\end{array}$ & $\begin{array}{l}\text { ply Iwist } \\
\text { factor }\end{array}$ & $\begin{array}{c}\text { Exp. } \\
\text { tenacity } \\
\text { g/lex }\end{array}$ & $\begin{array}{c}\text { cal. } \\
\text { Icuacity } \\
\text { g/tex }\end{array}$ \\
\hline $25.7 \times 2$ & 2715 & $\begin{array}{l}2025 \\
2707 \\
3130 \\
3546 \\
4088\end{array}$ & $\begin{array}{l}11.3 \\
135 \\
14.7 \\
15.2 \\
13.2\end{array}$ & $\begin{array}{l}9.5 \\
118 \\
131 \\
14.2 \\
138\end{array}$ & 7.8 & 71 & $1482 \times 3$ & 3123 & $\begin{array}{l}2136 \\
2594 \\
2968 \\
3365 \\
3809\end{array}$ & $\begin{array}{l}195 \\
218 \\
22.5 \\
227 \\
230\end{array}$ & $\begin{array}{l}16.1 \\
18.7 \\
20.3 \\
221 \\
219\end{array}$ \\
\hline $26.05 \times 2$ & 3354 & $\begin{array}{l}2337 \\
3291 \\
3596 \\
4180 \\
4784 \\
\end{array}$ & $\begin{array}{l}12.0 \\
14.3 \\
14.6 \\
135 \\
12.6\end{array}$ & $\begin{array}{c}9.7 \\
12.9 \\
13.5 \\
13.2 \\
126 \\
\end{array}$ & & & $14.93 \times 3$ & 3452 & $\begin{array}{l}2311 \\
2806 \\
3135 \\
3635 \\
3635 \\
\end{array}$ & $\begin{array}{ll}20 & 3 \\
21 & 5 \\
21.8 \\
225 \\
22 & 2\end{array}$ & $\begin{array}{l}165 \\
19.2 \\
206 \\
22.7 \\
21.6\end{array}$ \\
\hline $26.38 \times 2$ & 3791 & $\begin{array}{l}2605 \\
3519 \\
4230 \\
4610 \\
5260\end{array}$ & $\begin{array}{l}12.8 \\
143 \\
140 \\
131 \\
12.3\end{array}$ & $\begin{array}{l}10.1 \\
13.0 \\
12.9 \\
127 \\
12.0\end{array}$ & & & $1505 \times 3$ & 3916 & $\begin{array}{l}4087 \\
4492 \\
2533 \\
3011 \\
3437\end{array}$ & $\begin{array}{l}22.2 \\
21.0 \\
206 \\
21.0 \\
21.6\end{array}$ & $\begin{array}{l}21.6 \\
21.8 \\
17.0 \\
19.4 \\
21.1\end{array}$ \\
\hline $26.6 \times 2$ & 4352 & $\begin{array}{l}2863 \\
3587 \\
4415 \\
5249 \\
5978\end{array}$ & $\begin{array}{ll}13.4 \\
142 \\
13 & 1 \\
11 & 7 \\
11 & 1\end{array}$ & $\begin{array}{l}10.8 \\
125 \\
124 \\
11.8 \\
11.1\end{array}$ & & & $1512 \times 3$ & 4233 & $\begin{array}{l}4020 \\
4656 \\
5097 \\
2696 \\
3305 \\
3656 \\
4278 \\
4795 \\
5443\end{array}$ & $\begin{array}{lll}21 & .5 \\
20 & 1 \\
19 & 2 \\
21 & 0 \\
21 & 3 \\
21 & 5 \\
21 & 1 \\
19 & 5 \\
18 & 7\end{array}$ & $\begin{array}{l}21.5 \\
20.4 \\
19.8 \\
17.3 \\
201 \\
215 \\
20.7 \\
20.9 \\
189\end{array}$ \\
\hline
\end{tabular}




\begin{tabular}{|c|c|c|c|c|c|c|c|c|c|}
\hline $\begin{array}{l}\text { Tam roum } \\
\text { (lex) }\end{array}$ & $\begin{array}{l}\text { Single } \\
\text { Iwist } \\
\text { factor }\end{array}$ & $\begin{array}{l}\text { ply twist } \\
\text { faclor }\end{array}$ & $\begin{array}{c}\text { Exp. } \\
\text { tenocity } \\
\text { g/tex }\end{array}$ & $\begin{array}{c}\text { cal. } \\
\text { tenacily } \\
\text { g/tex }\end{array}$ & $\begin{array}{l}\text { Yarn } \\
\text { cunum } \\
\text { (tex) }\end{array}$ & $\begin{array}{l}\text { single } \\
\text { iwist } \\
\text { factar }\end{array}$ & $\begin{array}{l}\text { ply twist } \\
\text { factor }\end{array}$ & $\begin{array}{c}\text { Exp. } \\
\text { tewacily } \\
\text { g/lex }\end{array}$ & $\begin{array}{c}\text { Callenacity } \\
\text { g/tex }\end{array}$ \\
\hline \multirow[t]{6}{*}{$98 \times 3$} & \multirow[t]{6}{*}{2767} & 2272 & 163 & 17.9 & \multirow[t]{4}{*}{$11.45 \times 3$} & \multirow[t]{4}{*}{2593} & 1940 & 211 & 20.3 \\
\hline & & 2724 & 18.0 & 198 & & & 2472 & 237 & 23.9 \\
\hline & & 3010 & 20.5 & 20.6 & & & 3560 & 277 & 285 \\
\hline & & 3726 & 20.7 & 22.9 & & & 4119 & 261 & 279 \\
\hline & & 3937 & 203 & 22.5 & \multirow[t]{2}{*}{$11.65 \times 3$} & \multirow[t]{2}{*}{2980} & 2029 & 24.4 & 19.3 \\
\hline & & 4197 & 19.6 & 21.9 & & & 2784 & 26.9 & 24.3 \\
\hline \multirow{6}{*}{$\begin{array}{r}99 \times 3 \\
+\end{array}$} & \multirow[t]{6}{*}{3101} & 2610 & 17.3 & 189 & \multirow{6}{*}{$11.75 \times 3$} & \multirow{6}{*}{3462} & 4013 & 27.2 & 27.6 \\
\hline & & 2923 & 18.1 & 20.2 & & & 4650 & 26.0 & 26.3 \\
\hline & & 3308 & 19.7 & 21.0 & & & 2084 & 24.0 & 18.4 \\
\hline & & 4064 & 19.8 & 22.2 & & & 3143 & 27.9 & 25.1 \\
\hline & & 4291 & 19.9 & 21.3 & & & 3875 & 28.5 & 27.1 \\
\hline & & 4642 & 18.1 & 21.2 & & & 4495 & 25.3 & 25.8 \\
\hline \multirow[t]{6}{*}{$10 \times 3$} & \multirow[t]{6}{*}{3459} & 2910 & 182 & 19.3 & \multirow{5}{*}{$11.98 \times 3$} & \multirow{5}{*}{3946} & 5189 & $25 . \overline{0}$ & 24.4 \\
\hline & & 3364 & 19.3 & 21.3 & & & 2314 & 25.7 & 18.7 \\
\hline & & 3764 & 19.5 & 21.9 & & & 3447 & 27.4 & 25.5 \\
\hline & & 4360 & 18.8 & 21.0 & & & 4116 & 26.0 & 25.6 \\
\hline & & 4701 & 18.0 & 20.3 & & & 5770 & 22.8 & 22.3 \\
\hline & & 5075 & 173 & 198 & $12.2 \times 3$ & 4432 & 2559 & 24.8 & 196 \\
\hline \multirow[t]{6}{*}{$10.2 \times 3$} & \multirow[t]{6}{*}{4287} & 3422 & 18.5 & 20.2 & & & 3798 & 26.4 & 25.4 \\
\hline & & 3764 & 19.0 & 20.8 & & & 4702 & 25.7 & 242 \\
\hline & & 4503 & 18.3 & 19.9 & & & 6347 & 25.7 & 21.1 \\
\hline & & 5259 & 180 & 18.7 & & & & & \\
\hline & & 5548 & 17.7 & 183 & & & & & \\
\hline & & 5989 & 167 & 17.5 & & & & & \\
\hline
\end{tabular}


T77 I. M. Rakha
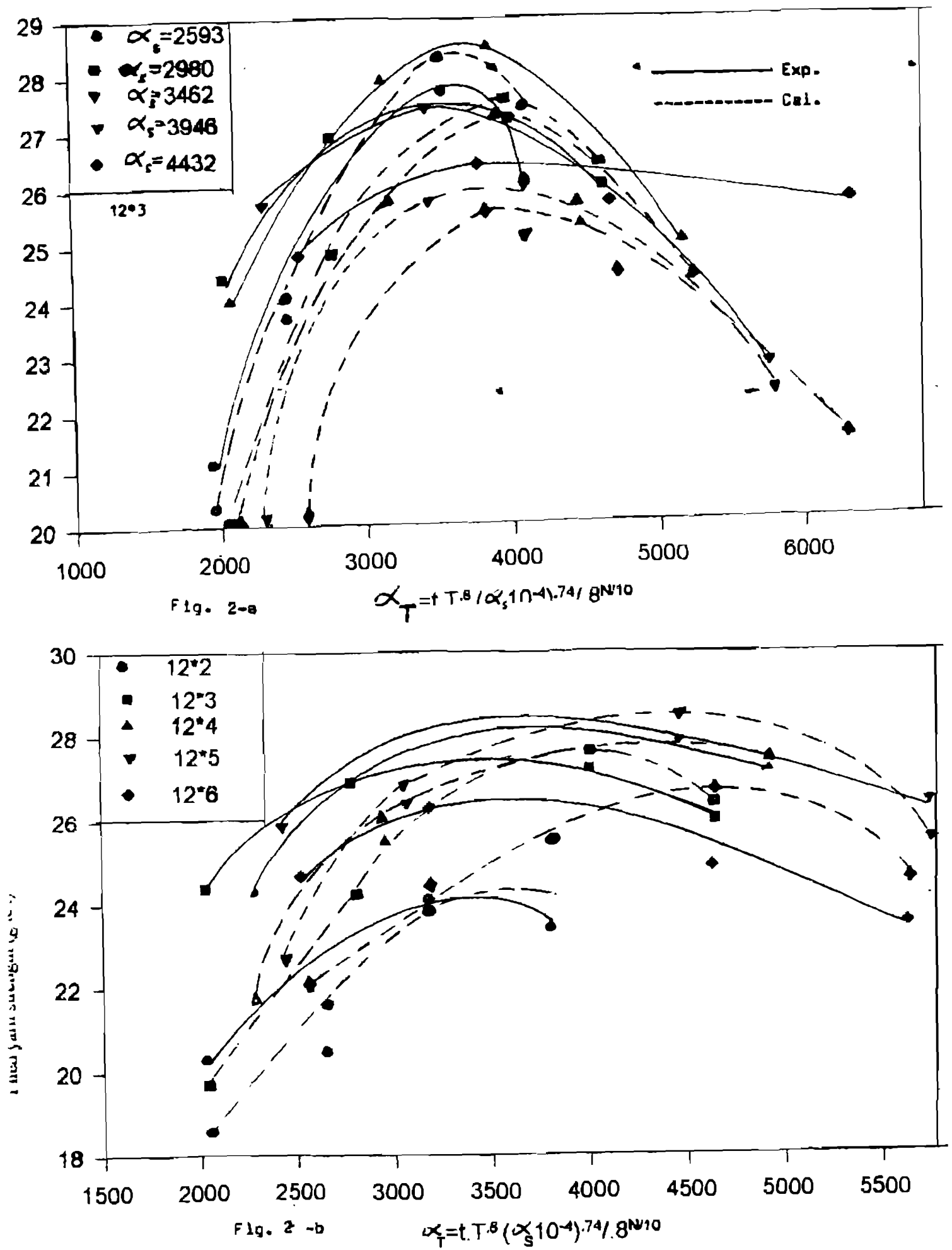